\title{
LED-Based Projection Systems
}

\author{
Xing-Jie Yu, Y. L. Ho, L. Tan, Ho-Chi Huang, and Hoi-Sing Kwok, Fellow, IEEE
}

\begin{abstract}
A novel design methodology for LED-array-based projection displays has been developed. By combining etendue limitation, system intensity, and efficiency requirements, a novel parameter space is proposed. Using this parameter space, LED lens-array and compound parabolic concentrator (CPC)-array illumination systems have been designed. A 1000-lm LED light source is built. Based on these lens-array and CPC-array illuminators, several LED-based liquid crystal on silicon (LCOS) projection systems are suggested. Among them, a one-panel LCOS projection system is proposed and tested. The method discussed here should be useful in the design of LED-array illumination systems for projectors in general.
\end{abstract}

Index Terms-LED arrays, projectors.

\section{INTRODUCTION}

$\mathbf{T}$ HE system performance of a light-valve projector is very much dependent on the characteristics of the illumination system, which includes the projection lamp and its associated optics (mainly the light collection system). A number of the illumination system characteristics can have significant effects on the performance of the projection system. Among these are: lamp power, lamp efficacy, lamp spectrum, and illumination system etendue (or F/\#). Other factors, such as lamp lifetime and warm-up time, can also influence the quality of the projector.

Currently, there are two major types of projection lamps used for light-valve projection systems: tungsten-halogen lamps and high-intensity discharge (HID) lamps that include metal-halide lamps, UHP lamps, and xenon lamps [1]. Tungsten-halogen lamps are incandescent lamps with halogen atoms incorporated in the gaseous fill surrounding the filament. Light is emitted from the tungsten filament at around $2800 \mathrm{~K}$ to $3200 \mathrm{~K}$. The advantages of these lamps are radiant cooling and low cost (only $0.0005 \$ /$ lumen). The disadvantages are low efficiency $(15 \mathrm{~lm} / \mathrm{W})$ and short lifetime. Also, the fundamental drawback is that it cannot achieve daylight color (color temperature $6500 \mathrm{~K}$ ) due to the lack of blue light in the spectrum. Thus, these lamps are not used in high-performance high-brightness projection systems. The HID lamp, also known as the arc lamp, consists of a sealed envelope containing a high-pressure gas and two electrodes. The fill gas can be xenon. A liquid such as mercury or a solid such as metal-halide salt is usually added to increase the efficiency. These lamps have very high efficiency $(100 \mathrm{~lm} / \mathrm{W})$, and the correlated color temperature (CCT) can be as high as $8500 \mathrm{~K}$. The major drawbacks are that they are quite

Manuscript received July 30, 3006; revised September 12, 2006. This work was supported by the Hong Kong Government Innovation and Technology Fund.

The authors are with the Center for Display Research, Department of Electrical and Electronic Engineering, Hong Kong University of Science and Technology Kowloon, Hong Kong (e-mail: eekwok@ust.hk).

Digital Object Identifier 10.1109/JDT.2007.901560 expensive and they produce strong UV/IR emissions. As well, mercury-containing lamps are environmentally unsuitable. Arc lamps are also dangerous as the glass envelope and electrodes can break easily. Despite these drawbacks, arc lamps are used widely in high-brightness projection systems.

As the projection market is increasing, alternative light sources are being explored. There is also a demand for portable low-cost projection systems which do not require very high brightness. Optical performance, portability, and low cost are crucial. HID lamps can provide good optical performance, but they are very expensive and not portable. Tungsten-halogen lamps satisfy the requirement on the cost, but the luminous efficiency and the color-rending ability are unacceptable for most applications. Thus, a novel illumination system needs to be developed for projection systems to satisfy all requirements: low cost, good color rendering, high luminous efficiency, and long lifetime.

Light-emitting diode (LED) technology is ideally suited for these applications [2]-[4]. It has the advantages of excellent color gamut $(>95 \% \mathrm{NTSC})$, long lifetime $(>50 \mathrm{~K} \cdot \mathrm{h})$, high brightness ( $>10 \mathrm{~K} \cdot \mathrm{nit})$, and ease of control and is environmentally friendly. The controllability leads to novel ideas such as blinking light source and field sequential color. The continuously improved efficiency makes it one of the best candidates for illumination systems [4]. For the case of projection light sources, generally an LED array has to be used. There have been proposals of single-chip LED projectors as well as LED arrays for high luminous projectors with 400-lm output [2], [3]. For such LED light sources, details, such as illumination optics and LED packaging for heat dissipation, have to be investigated thoroughly.

In this paper, we shall first develop a novel method on LED array optical design for projection systems. Based on this method, the LED lens-array and compound parabolic concentrator (CPC)-array illumination system will be designed and developed, respectively. The package for the LED array will be also studied. Then, several possible architectures on LED-based liquid crystal on silicon (LCOS) projection systems will be suggested. As an illustration, a one-panel LCOS system will be tested experimentally, and the performance will be reported.

\section{OPTICS OF LED ARRAYS}

The fundamental questions (or requirements) in any illumination system are: etendue or $\mathrm{F} / \#$ of the light source, light flux needed, and the acceptable light efficiency. The solution to the first requirement is given by the etendue limit [1]. If the area of the LED light source is $A_{\text {LED }}$ with the averaging emitting solid angle of $\Omega_{\mathrm{LED}}$, the etendue ( $E_{\mathrm{LED}}$ ) of this light source can be written as

$$
E_{\mathrm{LED}}=A_{\mathrm{LED}} \Omega_{\mathrm{LED}}
$$




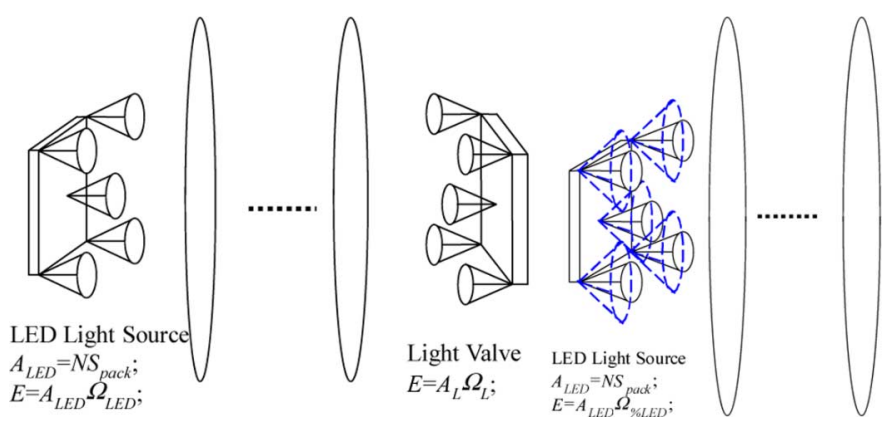

Fig. 1. Illumination system with $100 \%$ and $\eta_{\text {LED }}$ light utilization efficiency.

The image of this light source through the light collection system at the position of the light valve has the total area of $A_{\mathrm{I}}$ and an averaging solid angle of $\Omega_{I}$. For any optical systems, the etendue never decreases. Thus, the size of the light source is given by

$$
A_{\mathrm{LED}} \leq \frac{\Omega_{I}}{\Omega_{\mathrm{LED}}} A_{I}
$$

For the desired light collection system, as shown in Fig. 1(a), the total light flux of the LED source is completely projected onto the light valve with the requested $\mathrm{F} / \#$, that is, the area of $A_{I}$ is equal to the area of the light valve $A_{L}$. As well, the solid angle $\Omega_{I}$ should be equal to or less than the requested solid angle $\Omega_{L}$ for the light valve. Since the LED source should be compact, and the total area of LED light source is given, then

$$
A_{\mathrm{LED}}=N \bullet A_{\mathrm{pack}}
$$

where $N$ is the number of LEDs in the array and $A_{\text {pack }}$ is the area of each LED package. Thus, the LED maximum number $N$ is given by

$$
N \leq \frac{\Omega_{I}}{\Omega_{\mathrm{LED}}} \frac{A_{I}}{A_{\mathrm{pack}}} \leq \frac{\Omega_{L}}{\Omega_{\mathrm{LED}}} \frac{A_{L}}{A_{\mathrm{pack}}} .
$$

For the case described by (4), the illumination system has the highest light utilization efficiency (100\%), which means all of the light from the light source can be utilized.

If the LED number is increased, the total flux of the LED source will increase as well. However, at the same time, the light utilization efficiency will decrease since the etendue will be larger. The question is whether the flux of the light that can enter the light engine with the desired F/\# also increases.

Assume that light flux $\Phi_{\text {LED }}$ can enter the light engine and be utilized completely, as shown in Fig. 1(b). This flux $\Phi_{\mathrm{LED}}$ is contained in a solid angle of $\Omega_{\% \text { LED }}$. The light utilization efficiency is

$$
\eta_{\mathrm{LED}}=\frac{\Phi_{\mathrm{LED}}}{\Phi_{\mathrm{LED}-\text { total }}}
$$

where $\Phi_{\text {LED-total }}$ is the total flux of the single LED. Then, the maximum LED number $N$ for the illumination system with the utilization efficiency of $\eta_{\text {LED }}$ is given by

$$
N \leq \frac{\Omega_{L}}{\Omega_{\% \text { LED }}} \frac{A_{L}}{A_{\text {pack }}}
$$

which is similar to (4) except for the new solid angle $\Omega_{\% \text { LED }}$. The maximum usable light flux $F$ is

$$
\begin{aligned}
F & \leq \frac{\Omega_{L}}{\Omega_{\% \text { LED }}} \frac{A_{L}}{A_{\mathrm{pack}}} \eta_{\mathrm{LED}} \Phi_{\mathrm{LED}-\text { total }} \\
& =\left(\frac{\eta_{\mathrm{LED}}}{\Omega_{\% \mathrm{LED}} A_{\mathrm{pack}}}\right) \Omega_{L} A_{L} \Phi_{\mathrm{LED}-\text { total }}
\end{aligned}
$$

It should be noted that the calculations above are based on the estimated etendue given by (1). The more accurate etendue calculation [1] is given by

$$
E=\iint \cos \theta d \Omega d A \text {. }
$$

This calculation strongly depends on each specified optical system. Generally, no analytical equation can be derived. The analysis based on the accurate etendue calculation is quite complicated and sometimes impossible. Thus, it only can be done qualitatively. The results of our estimated calculations can be very good initial designs for the LED light sources. This will be verified later.

Note that the etendue does not refer to the light intensity within the distribution of interest: it only refers to the distribution's geometric boundaries. Thus, the second and third requirements in the illumination design are related to flux and efficiency. If the efficiency of the system light engine is $\eta_{\mathrm{LE}}$, then the maximum final output $\Phi_{\text {on-screen }}$ on the screen will be

$$
\begin{aligned}
\Phi_{\text {on-screen }}= & \eta_{\mathrm{LE}} F \\
= & \eta_{\mathrm{LE}} \bullet\left(\frac{\eta_{\mathrm{LED}}}{\Omega_{\% \text { LED }} A_{\text {pack }}}\right) \\
& \times \Omega_{L} A_{L} \Phi_{\mathrm{LED}-\text { total }} .
\end{aligned}
$$

If the input power of each LED is $1 \mathrm{~W}$, then the system luminous efficiency $\gamma$ is

$$
\gamma=\frac{\Phi_{\mathrm{on}-\mathrm{screen}}}{N \cdot 1}=\Phi_{\mathrm{LED}-\text { total }} \eta_{\mathrm{LED}} \eta_{\mathrm{LE}}
$$

Rewriting (9) and (10) gives

$$
\begin{aligned}
\frac{\Phi_{\mathrm{On}-\text { screen }}}{\Phi_{\mathrm{LED}-\text { total }}} & =\left(\frac{\eta_{\mathrm{LED}}}{\Omega_{\% \mathrm{LED}} A_{\mathrm{pack}}}\right) \Omega_{L} A_{L} \eta_{\mathrm{LE}} \\
\eta_{\mathrm{LED}} & =\frac{\gamma}{\Phi_{\mathrm{LED}-\text { total }} \eta_{\mathrm{LE}}} .
\end{aligned}
$$

Equations (11) and (12) are the key rules for the LED-array based illumination system design. The factors $\Omega_{L} A_{L}, \eta_{\mathrm{LE}}$, and $\Phi_{\text {LED-total }}$ depend on the system requirements (panel size, $\mathrm{F} / \#$, and light engine efficiency) as well as the LED die itself ( $\left.\Phi_{\mathrm{LED}-\text { total }}\right)$, while $\eta_{\mathrm{LED}} / A_{\text {pack }} \Omega_{\% \mathrm{LED}}$ is dependent on the LED optical performance and design.

Based on these rules, any LED array designs can be carried out in a parameter space. The factor $\left(\left(\eta_{\mathrm{LED}}\right) /\right.$ $\left.\left(\Omega_{\% \text { LED }} A_{\text {pack }}\right)\right) \Omega_{L} A_{L} \eta_{\text {LE }}$ for different LED optical designs is plotted as a function of the LED light collection efficiency $\eta_{\text {LED }} \cdot\left(\Phi_{\text {on-screen }}\right) /\left(\Phi_{\text {LED-total }}\right)$ and $(\gamma) /\left(\Phi_{\text {LED-total }} \eta_{\text {LE }}\right)$ are calculated according to the system requirements, and they give the minimum requirements or the boundaries in the parameter space, as shown in Fig. 2. Thus, the solutions will be in the shaded region. Table I lists these two boundaries with the light 


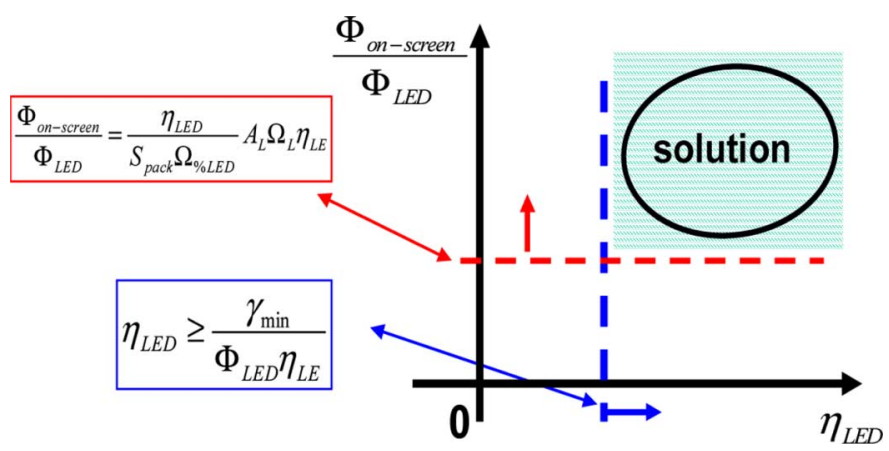

Fig. 2. Parameter space with minimum requirements or boundaries.

TABLE I

BOUNDARIES FOR DIFFERENT $\Phi_{\text {on-screen }}, \Phi_{\text {LED-total }}$ WITH $8 \% \eta_{\text {LE }}$

\begin{tabular}{|c|c|c|c|c|}
\hline$\eta_{L E}$ & \multicolumn{4}{|c|}{$8 \%$} \\
\hline$\Phi_{\text {on-screen }}$ & \multicolumn{2}{|c|}{$20 \mathrm{~lm}$} & \multicolumn{2}{c|}{$40 \mathrm{~lm}$} \\
\hline$\Phi_{\text {LED-total }}$ & $30 \mathrm{~lm}$ & $50 \mathrm{~lm}$ & $50 \mathrm{~lm}$ & $80 \mathrm{~lm}$ \\
\hline$\frac{\Phi_{\text {on-scren }}}{\Phi_{\text {LED-total }}}$ & 0.66 & 0.4 & 0.8 & 0.5 \\
\hline$\frac{\gamma}{\Phi_{L E D-\text { total }} \eta_{L E}}$ & $0.42 \gamma$ & $0.25 \gamma$ & $0.25 \gamma$ & $0.16 \gamma$ \\
\hline
\end{tabular}

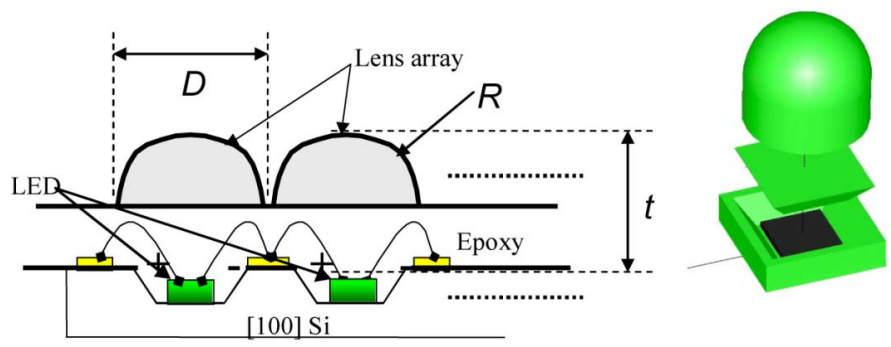

Fig. 3. Structure of LED lens array.

engine efficiency $\eta_{\mathrm{LE}}$ of $8 \%$ for different required final output $\Phi_{\text {on-screen }}$, the total flux $\Phi_{\text {LED-total }}$ of the single LED.

The objective of the LED illumination design is to find the LED optical package that satisfies these two minimum requirements. In this paper, the LED lens-array and CPC-array will be designed according to these laws.

The structure of the LED lens-array is shown in Fig. 3. The $1-\mathrm{W} 1-\mathrm{mm}^{2}$ LED dies are placed inside the wet-etched [100] $\mathrm{Si} \mathrm{V}$-grooves. On top of the LED-groove substrate, the lens array is then fabricated after wire bonding of the LED dies. The fabrication method for the lens array is a so-called "molding" method, which is originally developed for the micro-lens array used in the organic LEDs [5]. There are four physical parameters in the LED lens-array structure: the aperture $(D)$, the radius $(R)$, and the thickness $(t)$ and refractive index $(n)$ of the materials, as shown in Fig. 3. By varying these four parameters of the lens-array, $\left(\left(\eta_{\mathrm{LED}}\right) /\left(\Omega_{\% \mathrm{LED}} A_{\text {pack }}\right)\right) \Omega_{L} A_{L} \eta_{\mathrm{LE}}$ can be plotted as a function of the LED light collection efficiency $\eta_{\text {LED }}$. Take a 1 -mm radius $(R)$ silicone-based lens array $(n=1.49)$ as an example. In this case, only when $t \geq R$, light from the LED can be collected by this lens array efficiently.
Thus, $D_{\max }=2 R$ when $t \geq R$. Thus, the only variable is the lens-array thickness $(t)$. For each $t,\left(\eta_{\mathrm{LED}}\right) /\left(\Omega_{\% \mathrm{LED}} A_{\text {pack }}\right)$ can be easily calculated with commercial optical design software, such as TracePro, ASAP, or LightTools. Fig. 4 shows $\left(\left(\eta_{\mathrm{LED}}\right) /\left(\Omega_{\% \mathrm{LED}} A_{\text {pack }}\right)\right) \Omega_{L} A_{L} \eta_{\mathrm{LE}}$ versus $\eta_{\mathrm{LED}}$ parameter space as a function of the lens-array thickness $t$ with different $R$ (or $D$ ). Fig. 5 shows the maximum LED number $N$ based on (6) versus $\eta_{\text {LED }}$ parameter space as a function of the lens-array thickness $t$ with different $R$ (or $(D)$. The light valve used here is 0.87 -in LCOS panel with F/2 optics, and the light engine efficiency is $8 \%$.

According to the minimum requirements of $8 \%$ efficient LCOS systems listed in Table I, the boundaries in the parameter space are: 1) the flux boundary $\left(\Phi_{\text {on-screen }}\right) /\left(\Phi_{\text {LED-total }}\right) \geq 0.66$ and 2$)$ the efficiency boundary $\eta_{\mathrm{LED}}=(\gamma) /\left(\Phi_{\mathrm{LED}-\text { total }} \eta_{\mathrm{LE}}\right) \geq 0.42$ if the required final output is $20 \mathrm{~lm}$ with the system luminous efficiency of $1 \mathrm{~lm} / \mathrm{W}$ and assuming $30 \mathrm{~lm} / \mathrm{W}$ LED dies are used. Thus, the design solution in the parameter space should be above these two boundary planes. From Fig. 4, then the proper $t$ and $R(D)$ can be obtained (physical parameters of each LED optical structure). Once these parameters are known ( $D, R, t$ and $\eta_{\mathrm{LED}}$ ), from Fig. 5 , the number of LED $N$ can be found. For the lens-array solution, in order to achieve the final performance of $20-40 \mathrm{~lm}$ with the system luminous efficiency of $0.6-1.4 \mathrm{~lm} / \mathrm{W}, 15-40 \mathrm{LEDs}$ with $50 \mathrm{~lm} / \mathrm{LED}$ will have to be used. The questions on LED illumination design, including the structure of the lens arrays $(D, R, t)$, number of LEDs, and the light collection efficiency $\eta_{\mathrm{LED}}$, can be obtained in the parameter space.

The problem for LED lens-array method is that it needs many LEDs to achieve a large final output. This will result in a low system luminous efficiency, if the light engine efficiency is not high enough. The reason for this is that the lens array is not a perfect optical component for light collection.

In order to solve this problem, a solid CPC-array has been designed. The circular CPC was developed recently in an LED illumination system [6]. However, because the LED and the light valve (TFT, LCOS, and DLP) are all rectangular, the CPC should also be rectangular in order to obtain a high light collection efficiency $\eta_{\text {LED }}$. Our rectangular CPC and LED CPC-array structures $(2 \times 3)$ are shown in Fig. 6. There are five shape parameters for this CPC: focal length $f$ of the parabolic shape, tilt angle $\alpha$ of the parabolic axis, the front length FL, the back length BL, and focal shift DF. From a design point of view, the output diameter $\left(D_{\text {out }}\right)$ and the input diameter $\left(D_{\text {in }}\right)$ of a CPC is more important, and both are functions of the five shape parameters. The input diameter $\left(D_{\text {in }}\right)$ is limited by the size of LED dies and packaging of the LED dies. Thus, the main design parameters will be $D_{\text {out }}$ and the total length (LF-LB).

Similar to the lens-array design, by varying these five parameters of the CPC, the design parameters ( $D_{\text {out }}$ and LF-LB) can be calculated. For different design parameters, $\left(\left(\eta_{\mathrm{LED}}\right) /\left(\Omega_{\% \text { LED }} A_{\text {pack }}\right)\right) \Omega_{L} A_{L} \eta_{\mathrm{LE}}$ can be plotted as a function of the LED light collection efficiency $\eta_{\text {LED }}$. This result is shown in Fig. 7 as a function of $D_{\text {out }}$ of the CPC. Fig. 8 shows the maximum LED number $N$ based on (6) versus $\eta_{\text {LED }}$ parameter space as a function of the output diameter of the CPC. 

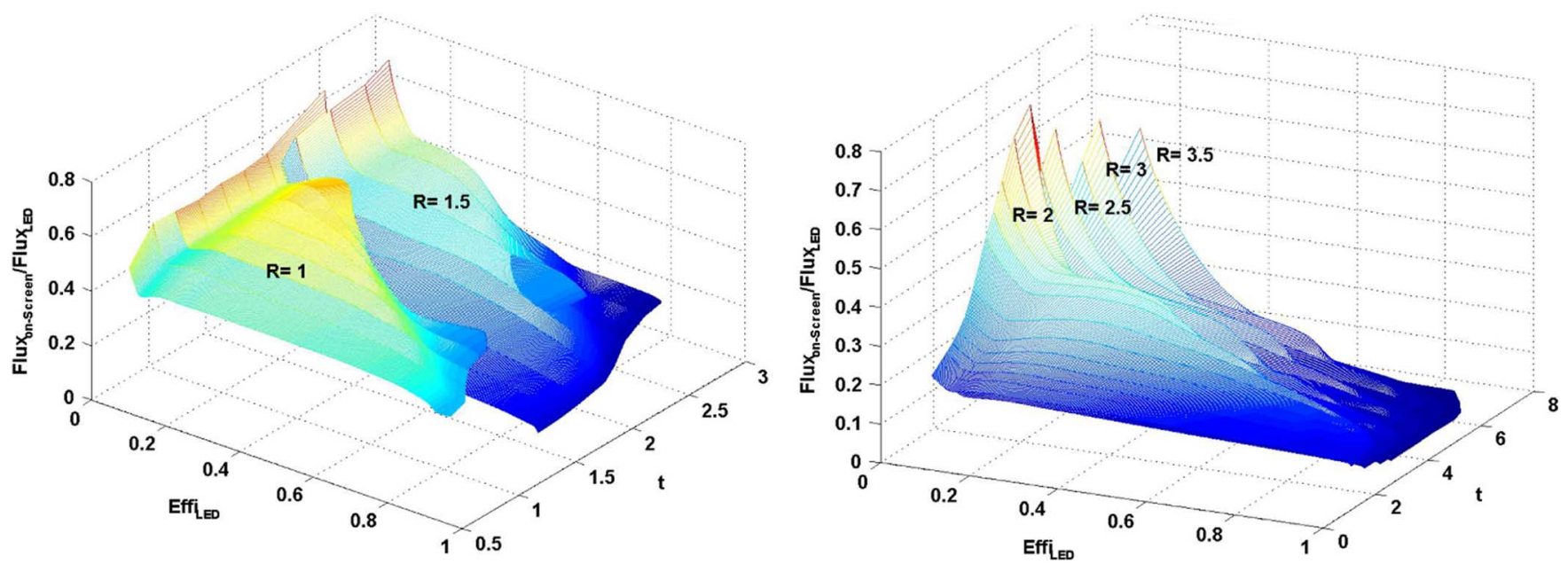

Fig. 4. $\left(\left(\eta_{\mathrm{LED}}\right) /\left(\Omega_{\% \mathrm{LED}} A_{\text {pack }}\right)\right) \Omega_{L} A_{L} \eta_{\mathrm{LE}}$ versus $\eta_{\mathrm{LED}}$ parameter space for the lens array.
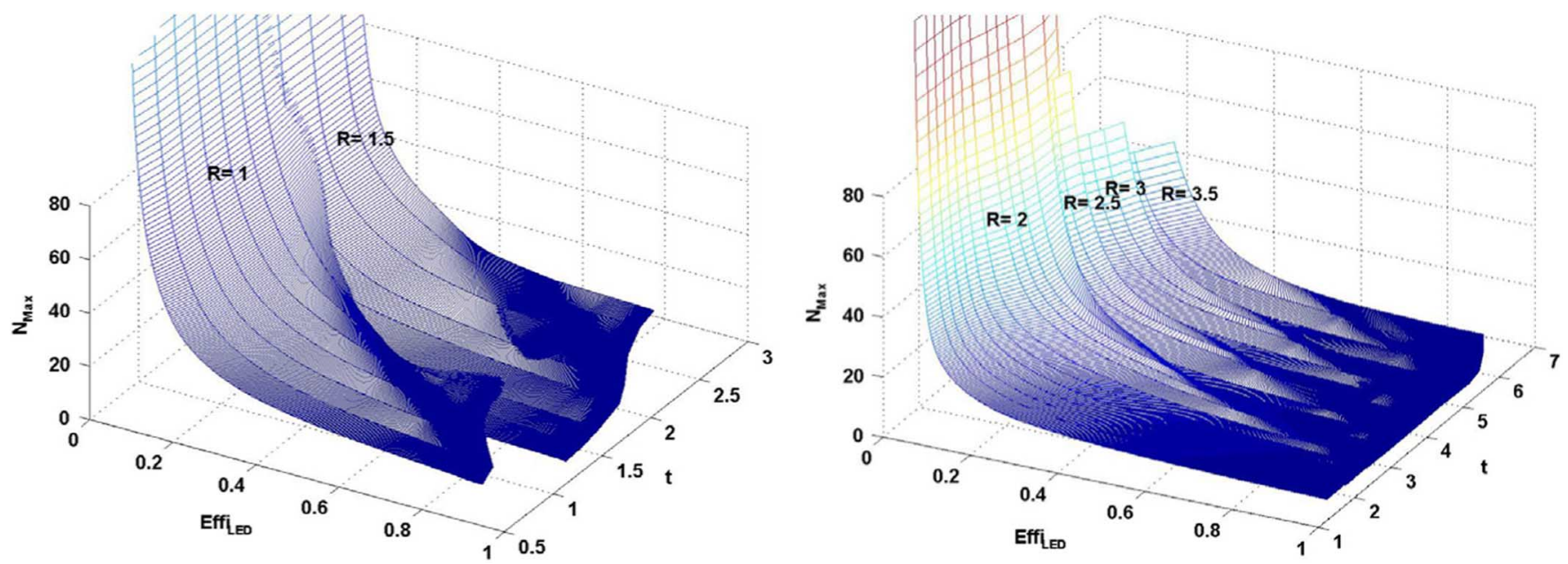

Fig. 5. Maximum LED number $N$ versus $\eta_{\text {LED }}$ parameter space for the lens array.

Compared with the lens-array parameter space, for the same boundary (e.g., $\left(\Phi_{\text {on-screen }}\right) /\left(\Phi_{\text {LED-total }}\right) \geq 0.66 ; \eta_{\text {LED }}=$ $\left.(\gamma) /\left(\Phi_{\text {LED-total }} \eta_{\text {LE }}\right) \geq 0.42\right)$, the CPC system has solutions with high system luminous efficiency. For the same engine system and a final output of $20 \mathrm{~lm}$, the CPC needs only six LEDs with an overall system luminous efficiency of $>3 \mathrm{~lm} / \mathrm{W}$.

\section{LED ILLUMINATION SYSTEM}

\section{A. Packaging}

After the optical design of the LED array is done, the LED illumination system can be packaged. Although the lens-array system has lower efficiency than the CPC one, we still use it in our investigation due to the ease of fabrication. According to the lens-array design, the LED has to be packaged very close to each other. The most important issue for this ultracompact LED light source is heat dissipation. A $1-\mathrm{mm}^{2}$ LED dissipating $1 \mathrm{~W}$ corresponds to $100 \mathrm{~W} / \mathrm{cm}^{2}$ of heat flux, which is twice the amount of the heat flux generated in a conventional microprocessor chip. Therefore, the effective removal of heat (to maintain a safe junction temperature) is the key to LED light source package. Nowa- days, flip-chip technology (FCT) is one of the best solutions for heat dissipation. It can be used in our package.

We also developed a normal package for the LED lens array. The idea is that, using a silicon wafer as the substrate, heat can be dissipated efficiently due to its high thermal conductivity. The LED dies are placed on the bottom of the wet-etched [100] silicon V-shape groove as shown in Fig. 3. The surfaces of these V-shape grooves are coated with a metal such as aluminum, which serves as a mirror. The shape of the $\mathrm{V}$-shape groove is important optically. Then, the lens array with the desired dimension based on our design is formed on top of the optimal LED-groove substrate. The LED dies are mounted on the $\mathrm{V}$-groove substrate by using thermally conductive epoxy. The same epoxy is also used to glue the silicon substrate onto the aluminum heat sink.

The heat flow through the conducting layers attached in parallel or series can be analyzed in the equivalent thermal circuit [7]. Thermal conductivities of the various materials used in our package must be maximized. Of particular interest are the various interface materials, which constitute a large portion of the thermal resistance. In most cases, contact resistances rather than the bulk resistance of the interface material dominate the overall 

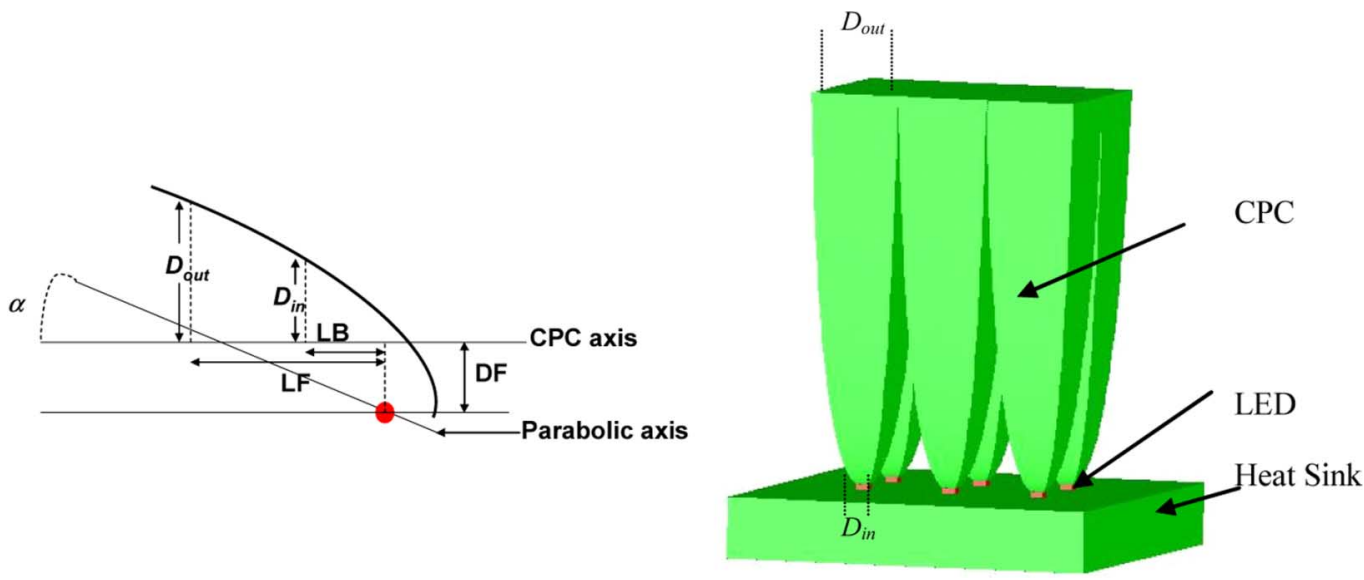

Fig. 6. Rectangular CPC and LED CPC-array structures.

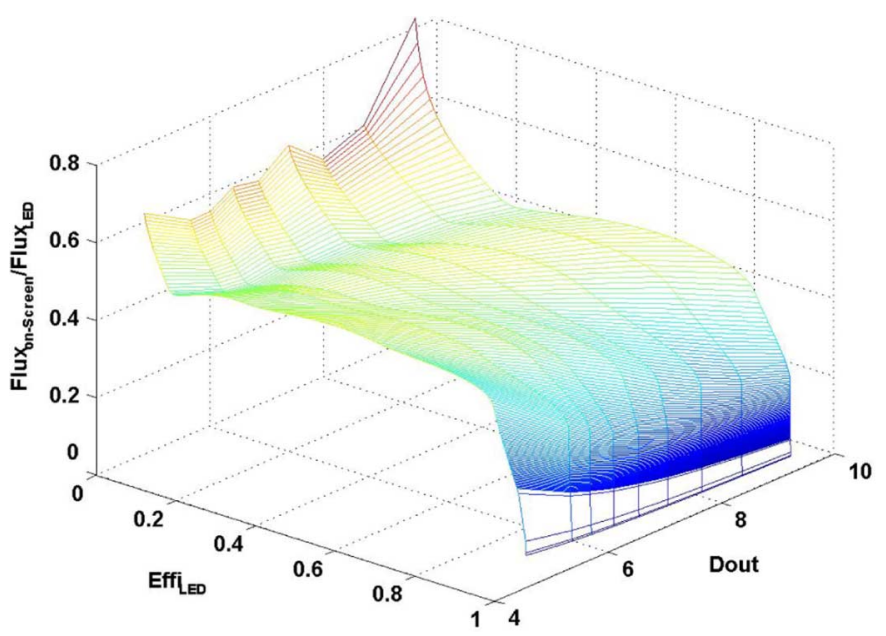

Fig. 7. $\left(\left(\eta_{\mathrm{LED}}\right) /\left(\Omega_{\% \text { LED }} A_{\mathrm{pack}}\right)\right) \Omega_{L} A_{L} \eta_{\mathrm{LE}}$ versus $\eta_{\mathrm{LED}}$ parameter space for the CPC array.

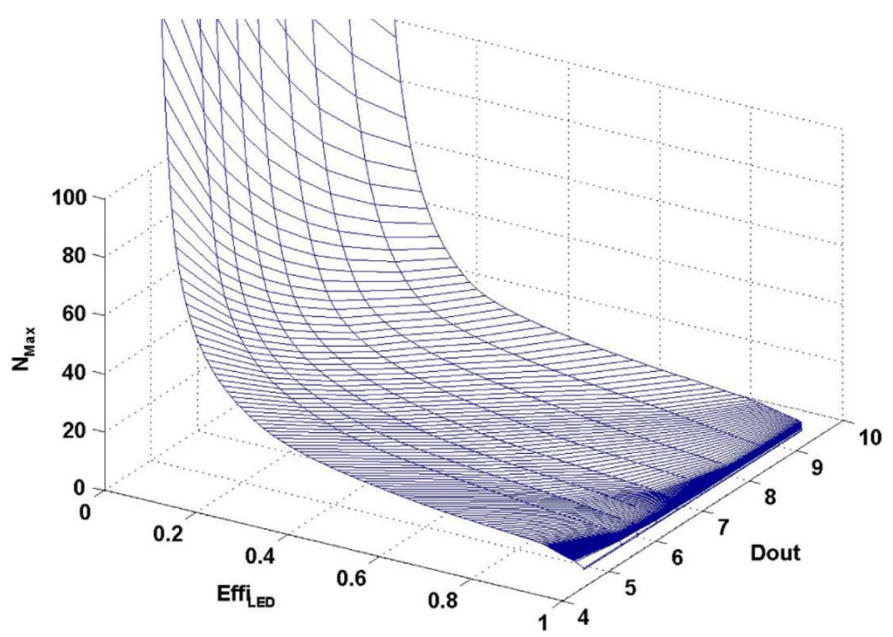

Fig. 8. Maximum LED number $N$ versus $\eta_{\text {LED }}$ parameter space for the CPC array.

thermal resistance. By selecting the epoxy at the interfaces and other package materials, the overall thermal resistance of our

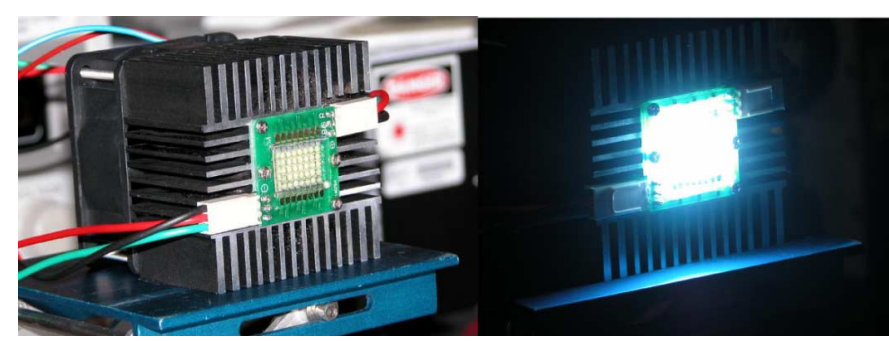

Fig. 9. LED lens-array light source package with 40 1-W LEDs.

LED lens-array package is only limited by the LED sapphire substrate, which is $30^{\circ} \mathrm{C} / \mathrm{W}$. The significant decrease in thermal resistance (compared with the traditional 5-mm package $\mathrm{T}-1$ and T-1 $3 / 4, R_{\text {thjp }}>200^{\circ} \mathrm{C} / \mathrm{W}$ ) allows high-current-density operation.

It is estimated that the maximum junction temperature for a $1-\mathrm{mm}^{2} \mathrm{LED}$ die is around $120^{\circ} \mathrm{C}$. The measured package temperature is $<40{ }^{\circ} \mathrm{C}$ for 401 -W LEDs packaged at the area of $160 \mathrm{~mm}^{2}$. Thus, the permissible dissipation of our package is approximately $2 \mathrm{~W}$. Fig. 9 shows the LED lens-array light source with $40(8 \times 5) 1$-W LEDs (blue LEDs plus yellow phosphor) packaged at the area of $160 \mathrm{~mm}^{2}$. The bare LED chips are from Epistar Corporation, with 27-30 lm/LED. The total output of this light source is $1000-1100-1 \mathrm{~m}$ white light at a driving current of $350 \mathrm{~mA}$ for each LED. The R, G, and B LEDs are also tested on our package, and similar performance is obtained.

For the CPC array solution, the LED package is much easier since less LEDs are used and LEDs are placed several millimeters ( $\sim 7 \mathrm{~mm}$ in our design) away from each other. Thus, both FCT and our normal package can be used, and the heat dissipation will not be a problem. We also package the CPC system with a $2 \times 3$ LED array.

\section{B. Polarization Conversion}

If the LED illumination system is employed for the projection system based on a liquid crystal light valve, polarization conversion is needed [8]-[12]. The simple reflective polarizer and the broadband quarterwave plate (QWP) can be used as the polarization converter for our LED source, as illustrated in Fig. 10, 

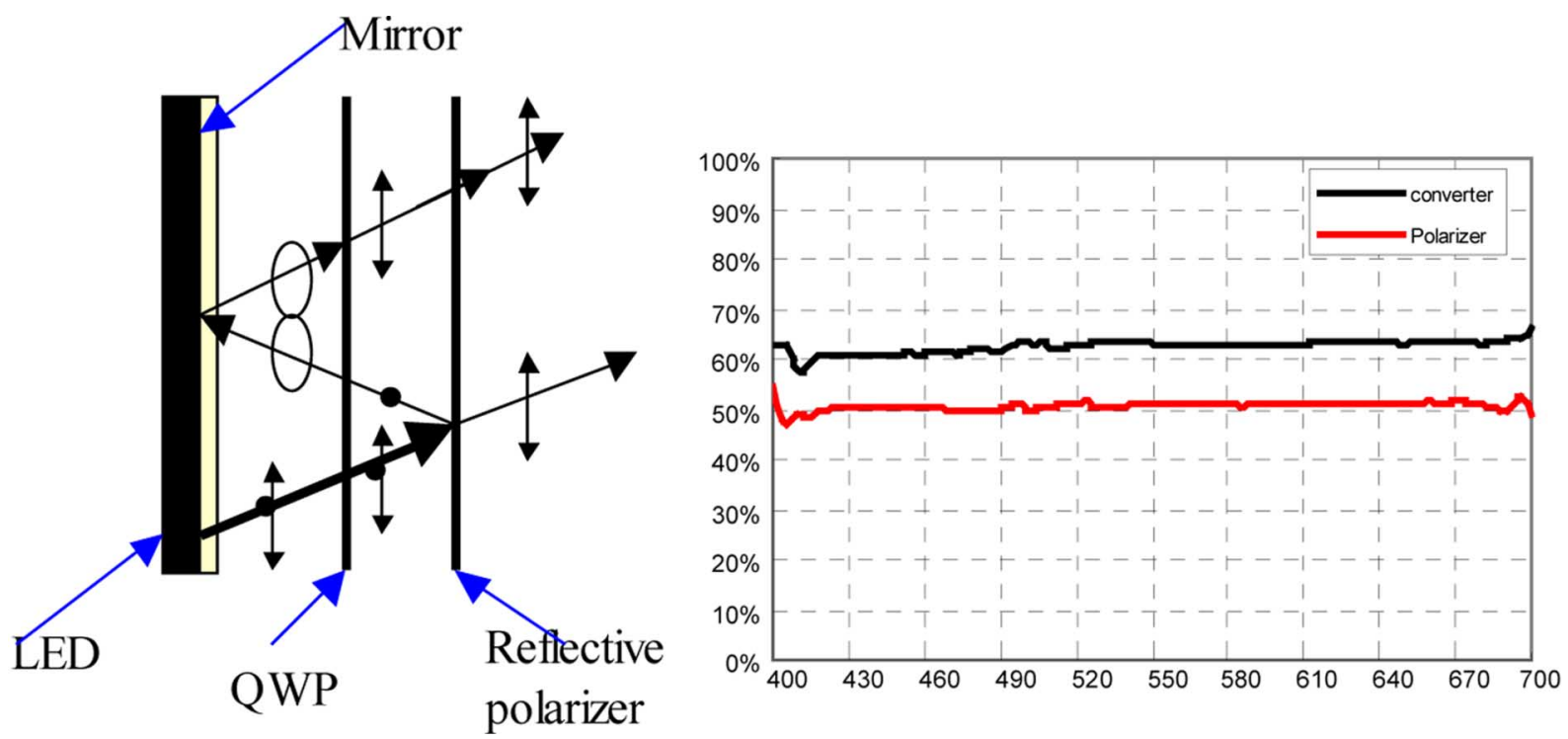

Fig. 10. Structure of the polarization converter and its polarization conversion efficiency.

TABLE II

LIGHT COLLECTION EFFICIENCY $\eta_{\text {LED }}$ FOR SEVERAL $2 \times 3$ CPC-ARRAY SYSTEMS

\begin{tabular}{|c|c|c|c|c|c|c|c|c|c|c|c|c|}
\hline CPC & \multicolumn{3}{|c|}{$4.9 \mathrm{~mm}\left(\boldsymbol{D}_{\text {out }}\right)$} & \multicolumn{5}{|c|}{$6.4 \mathrm{~mm}\left(\boldsymbol{D}_{\text {out }}\right)$} & \multicolumn{4}{|c|}{$9.5 \mathrm{~mm}\left(\boldsymbol{D}_{\text {out }}\right)$} \\
\hline Lens number & 0 & 1 & 2 & Ideal & 0 & 1 & 2 & Ideal & 0 & 1 & 2 & Ideal \\
\hline 0.6 inch & $22 \%$ & $30 \%$ & $33 \%$ & $38 \%$ & $27 \%$ & $30 \%$ & $33 \%$ & $36 \%$ & $18 \%$ & $33 \%$ & $35 \%$ & $37 \%$ \\
\hline 0.87 inch & $39 \%$ & $62 \%$ & $79 \%$ & $85 \%$ & $54 \%$ & $63 \%$ & $78 \%$ & $85 \%$ & $47 \%$ & $65 \%$ & $80 \%$ & $85 \%$ \\
\hline
\end{tabular}

which also shows its experimental polarization conversion efficiency. The single reflective polarizer has $50 \%$ conversion efficiency, obviously. The efficiency for our polarization converter is around $64 \%$.

\section{Light Collection from LED Light Sources}

The LED light collection efficiency $\eta_{\text {LED }}$ obtained from the parameter space is the maximum value that can be achieved, which is calculated according to the etendue law. In the practical system, the simplest way to achieve this value is to use condenser lenses. We have done the optimizations on the condenser lenses for both lens-array and CPC-array systems. Table II summarizes the real light collection efficiency $\eta_{\mathrm{LED}}$ by using zero, one, and two condenser lenses for several CPC-array systems, compared with the ideal value from our parameter space method, for different panel sizes (0.6 and $0.86 \mathrm{in}$ ). For example, for $D_{\text {out }}$ of $6.4-\mathrm{mm} \mathrm{CPC}$, from our parameter space estimation, a $2 \times 3 \mathrm{CPC}$ array will be corresponding to $36 \%$ light collection efficiency for a $0.6 \mathrm{LCOS}$ panel. By using a different number of condenser lenses, the maximum practical efficiency can be $33 \%$. Thus, it can be seen that the real efficiency is close to the ideal one by using two condenser lenses. Sometimes, even one condenser lens is acceptable for the simple system configuration. In our practical systems, either one or two general BK7 condenser lenses are employed depending on the panel size used.

\section{LED-BASEd LCOS PROJECTION SySTEMS}

Based on our optical designs and packages on LED illumination systems, several LED-based LCOS projection systems are suggested. In these systems, a 0.87 -in $1280 \times 768$ color filter (CF) LCOS panel is used as the LCOS light valve [13]. ${ }^{1}$ Fig. 11 shows the LED-based one-panel system using either blue LED and yellow phosphor or RGB-LED combination. For the RGB-LED combination, they can be separated onto three blocks as shown. The red-, green-, and blue-LED light sources can be combined with an X-cube. Since the red LEDs have the highest luminous efficiency and the blue light is not very important in the projected images, the red and blue LEDs can also be placed on the same block in a two-block system, as shown in Fig. 11(b). This will simplify the optical design somewhat.

Table III summarizes the expected final outputs of one-panel systems based on current LED technologies (late 2005), according to our optimization on LED illumination system from the parameter space method. In this calculation, $\eta_{\mathrm{LE}}$ is assumed to be $8 \%$. With more efficient LEDs, this table needs to be modified accordingly. This modification should be rather straightforward.

It can be seen that the LED-based one-panel system can provide the final output of $15-40 \mathrm{~lm}$. The blue LED plus yellow phosphor combination is simple and low-cost. Based on this solution, the final output of $20 \mathrm{~lm}$ is still good for the application where the projected screen diagonal is smaller than $10 \mathrm{in.} \mathrm{For}$ $2 \times$ more efficient LED, which is available currently, the final projector output will be more than $2 \times$, since a smaller number of LED can be used, which improves the etendue requirement. It is estimated that the final output can be more than $3 \times$ or $120 \mathrm{~lm}$.

One of our targets is a 10-in LED-based mini-projector. Fig. 12 shows our practical LED lens-array system setup for this target. The 40-LED light source demonstrated above is applied to this system integration. The total flux of our 40-LED

\footnotetext{
${ }^{1}$ Color-filter LCOS (CF-LCOS) panels are obtained from Integrated Microdisplays Limited (iMD) of Hong Kong. Descriptions of CF-LOCS panels can be found in iMD website www.hkimd.com
} 


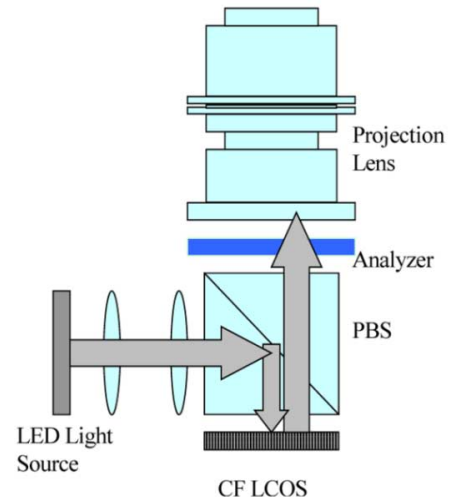

(a)

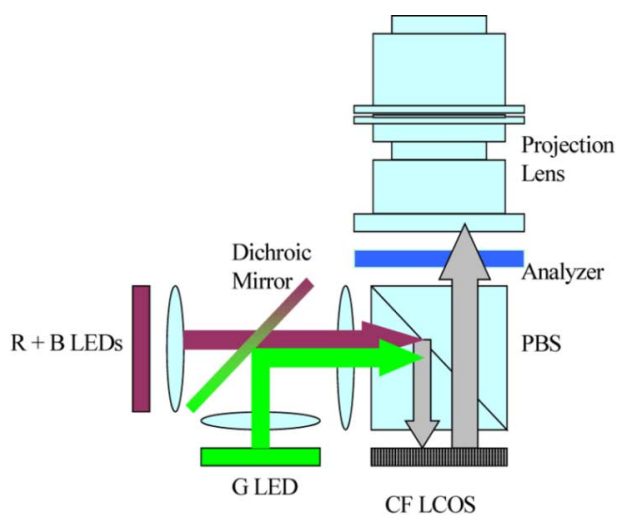

(b)

Fig. 11. LED-based one-panel system.

TABLE III

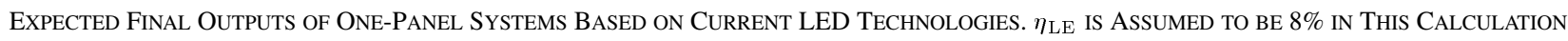

\begin{tabular}{|c|c|c|c|c|c|}
\hline Array & LED Technology & $\Phi_{L E D \text {-total }}$ & $\begin{array}{c}\text { LED } \\
\text { Number/Chip }\end{array}$ & $\eta_{\text {LED }}$ & $\begin{array}{l}\text { Projector Output } \\
(\mathrm{lm})\end{array}$ \\
\hline \multirow{4}{*}{$\begin{array}{l}\text { lens } \\
\text { array }\end{array}$} & Blue LED+ Phosphor & 50 & 40 & $15 \%$ & 24 \\
\hline & Blue LED+ Phosphor & 50 & 15 & $37 \%$ & 22 \\
\hline & $\begin{array}{c}\text { RGB-LED + Dichroic } \\
\text { Mirror }\end{array}$ & $\begin{array}{l}\text { R: G: B } \\
50: 50: 16\end{array}$ & 40 & $15 \%$ & 40 \\
\hline & $\begin{array}{c}\text { RGB-LED + Dichroic } \\
\text { Mirror }\end{array}$ & $\begin{array}{l}\text { R: G: B } \\
50: 50: 16\end{array}$ & 15 & $37 \%$ & 37 \\
\hline \multirow{4}{*}{$\begin{array}{l}\mathrm{CPC} \\
\text { array }\end{array}$} & Blue LED+ Phosphor & 50 & 6 & $85 \%$ & 20 \\
\hline & Blue LED+ Phosphor & 50 & 12 & $50 \%$ & 24 \\
\hline & $\begin{array}{l}\text { RGB-LED+Dichroic } \\
\text { Mirror }\end{array}$ & $\begin{array}{l}\text { R: G: B } \\
50: 50: 16\end{array}$ & 6 & $85 \%$ & 34 \\
\hline & $\begin{array}{c}\text { RGB-LED+Dichroic } \\
\text { Mirror }\end{array}$ & $\begin{array}{l}\text { R: G: B } \\
50: 50: 16\end{array}$ & 12 & $50 \%$ & 40 \\
\hline
\end{tabular}
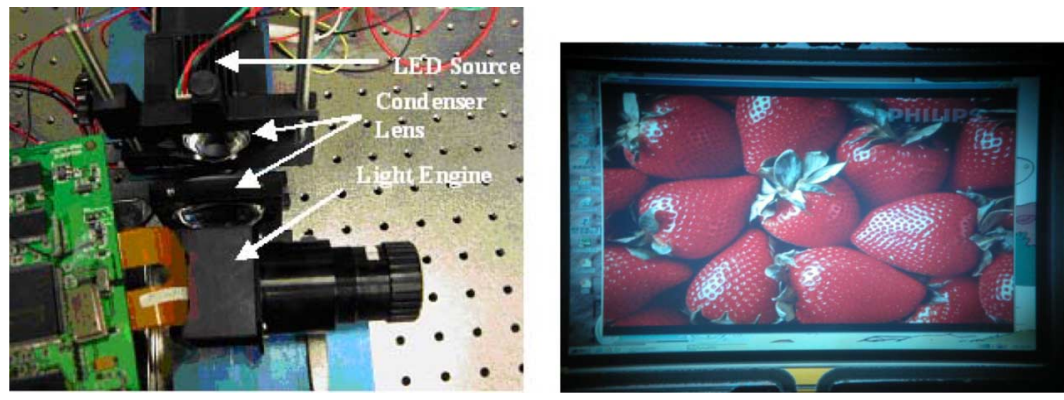

Fig. 12. Practical blue-LED + yellow-phosphor-based one-panel system and its projected image.

light source provides $>1000 \mathrm{~lm}$. The measured $\eta_{\text {LED }}$ is $15 \%$ for our present system. The practical $\eta_{\mathrm{LE}}$ is $8 \%$. So, the final output should be $12 \mathrm{~lm}$ according to our estimation based on (7.1) and the measured $\eta_{\mathrm{LE}}$ and $\eta_{\mathrm{LED}}$. The final experimental output is $11 \mathrm{~lm}$, which is consistent with our design value. The practical projected image of our LED-based one-panel projector is also shown in Fig. 12.

If more flux output and larger color gamut are requested, an RGB-LED light engine will have to be used. Fig. 13 shows the practical LED lens-array system for this target and its associated projected image. The medium-flux RGB LEDs are used in this system integration. This system has the potential to provide 40-lm output, as shown in Table III.

The LED lens-array system achieves the required target by using more LEDs corresponding to low system luminous efficiency, while an LED CPC-array system shows the same performance by using less than ten LEDs. In our demonstration, an $2 \times 3$ LED CPC-array is used. This system can provide $>20 \mathrm{~lm}$ output by just using six LEDs.

The final flux outputs of these two systems are still not high enough for the large-screen ( $>40$ in) rear projection television (RPTV) applications. If the LED light source is still used for 

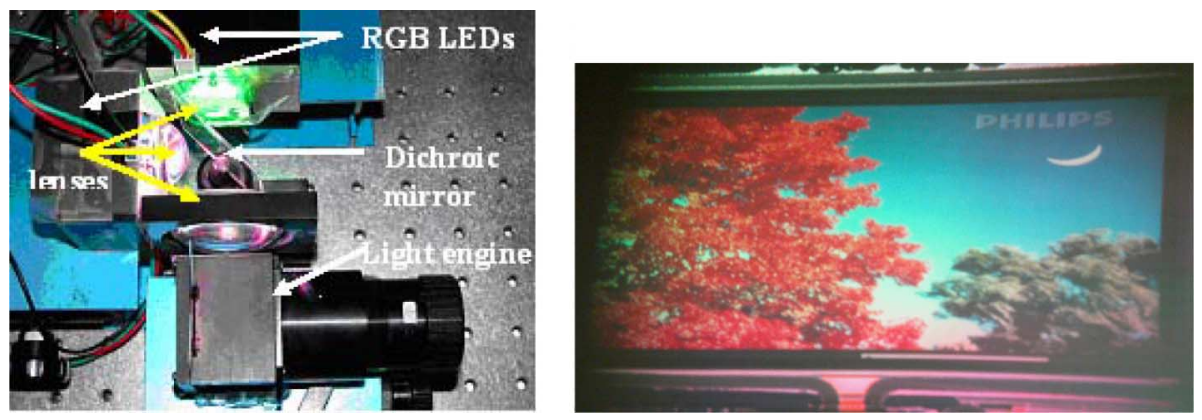

Fig. 13. Practical RGB-LED-based one-panel system and its projected image.
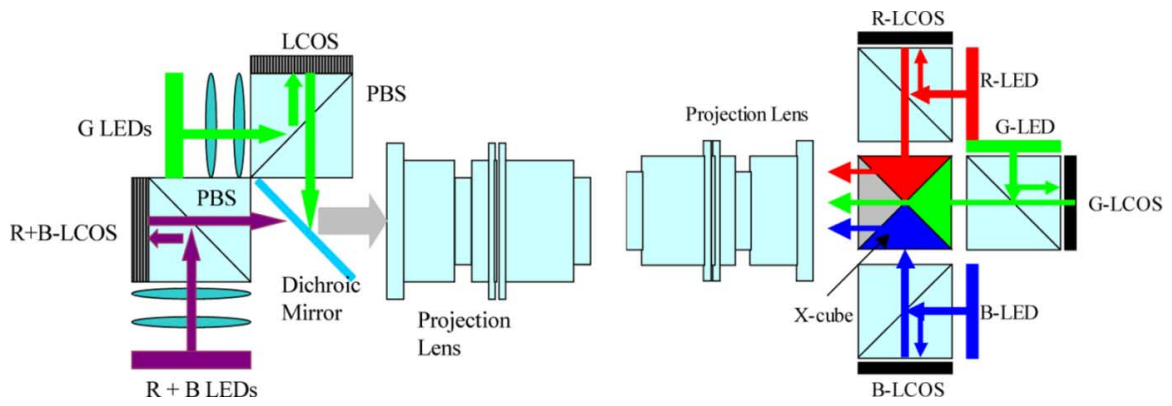

Fig. 14. Two-/three-panel system configurations.

TABLE IV

EXPECTED FINAL OUTPUTS OF TwO-/THREE-PANEL SySTEMS

\begin{tabular}{|c|c|c|c|c|c|}
\hline Array & LED Technology & $\Phi_{\text {LED-total }}$ & $\begin{array}{c}\text { LED } \\
\text { Number/Chip }\end{array}$ & $\eta_{\text {LED }}$ & $\begin{array}{c}\text { Projector Output } \\
(\mathrm{lm})\end{array}$ \\
\hline $\begin{array}{c}\text { lens } \\
\text { array }\end{array}$ & RGB-LED & G: $50 \mathrm{~lm}$ & 15 & $37 \%$ & 160 \\
\hline $\begin{array}{c}\text { CPC } \\
\text { array }\end{array}$ & RGB-LED & G: $50 \mathrm{~lm}$ & 6 & $85 \%$ & 145 \\
\cline { 2 - 6 } & RGB-LED & G: $50 \mathrm{~lm}$ & 12 & $50 \%$ & 170 \\
\hline
\end{tabular}

the large-screen RPTV application due to its good color rendering and low cost, then the LED-based two-/three-panel systems are suggested. The system configurations are shown in Fig. 14. These two systems have a similar efficiency, because the efficiency of the green light dominates the overall system efficiency and both systems have the same efficiency for the green light. Table IV summarizes the expected final outputs of the systems based on current LED technologies. In this calculation, $\eta_{\text {system }}$ is assumed to be $34 \%$. It can be seen that these two systems can provide $>150$-lm output that will be good for HDTV applications. Due to the cost and complexity of different systems, only a one-panel system is tested experimentally in this paper. Two-/three-panel systems based on a CPC array will be concentrated in future studies of HDTV applications.

\section{CONCLUSION}

A novel design method of an LED-array illumination system for projection displays has been developed. By combining etendue limitation, intensity, and efficiency requirements, a parameter space method is proposed. The factor $\left(\left(\eta_{\mathrm{LED}}\right) /\left(\Omega_{\% \mathrm{LED}} A_{\text {pack }}\right)\right) \Omega_{L} A_{L} \eta_{\mathrm{LE}}$ is plotted as a function of the LED light collection efficiency $\eta_{\text {LED }}$ with varying physical parameters of the different LED optical designs. According to the minimum requirements $\left(\left(\Phi_{\text {on-screen }}\right) /\left(\Phi_{\mathrm{LED}-\text { total }}\right)\right.$ and $\left.(\gamma) /\left(\Phi_{\mathrm{LED}-\text { total }} \eta_{\mathrm{LE}}\right)\right)$ in the above parameter space, various solutions can be obtained.

According to this law, the LED lens-array and CPC-array systems have been designed and developed. The optical performances and packages of these systems are examined. The final output $>1000$-lm LED light source is built. Based on the designs of the lens-array and CPC-array illumination systems, several LED-based LCOS projection systems are suggested.

Due to the cost and complexity of different systems, in this paper only a one-panel LCOS projection system is tested to prove our optical designs. The results show that our method is useful in the design of LED-array illumination systems. The output obtained agrees with that from both simulations and experiments.

\section{ACKNOWLEDGMENT}

The authors would like to thank Epistar Corporation for supplying the LED chips in this study.

\section{REFERENCES}

[1] E. H. Stupp and M. S. Brennesholtz, Projection Displays. New York: Wiley, 1998.

[2] M. H. Keuper, G. Harbers, and S. Paolini, "RGB LED illuminator for pocket sized projectors," Proc. Soc. Inf. Disp. (SID’04), p. 943, 2004.

[3] W. Folkerts, Proc. Soc. Inf. Disp. (SID'04), p. 1226, 2004. 
[4] Sandia, Solid-State Lighting Archived Headline News (May 11, 2004) [Online]. Available: http://lighting.sandia.gov/Xlightingnewsarchive.htm

[5] H. J. Peng, Y. L. Ho, X. J. Yu, M. Wong, and H. S. Kwok, "Coupling efficiency enhancement in organic light-emitting devices using microlens array-theory and experiment," J. Display Technol., vol. 1, no. 2, pp. 278-282, Dec. 2005.

[6] H. Zou, A. Schleicher, and J. Dean, "Single panel LCOS color projector with LED light sources," in Proc. Soc. Inf. Disp. (SID'05), 2005, vol. 36, p. 1698.

[7] N. Narendran, Y. Gu, and R. Hosseinzadeh, "Estimating junction temperature of high-flux white LEDs," Proc. SPIE, vol. 5366, pp. 158-160, 2004.

[8] C. Nicolas, B. Loiseaux, and J. P. Huignard, "Polarized light source for LCD projection,” Displays, vol. 16, p. 43, 1995.

[9] B. G. Rho and J. S. Kim, "A new LCD projection system with higher brightness," in Proc. 15th Int. Display Res. Conf., Hamamatsu, Japan, 1995 , p. 83.

[10] T. Gunjima, M. Ozeki, and Y. Ooi, "New back-lighting devices for liquid crystal displays," in Proc. 15th Int. Display Res. Conf., Hamamatsu, Japan, 1995, pp. 731-????.

[11] S. Shikama, E. Toids, and M. Kondo, "A polarization transforming optics for high luminance LCD projector," in Proc. Soc. Inf. Disp., 1991, vol. 32 , p. 301.

[12] A. deVaan, A. de Brandt, R. Karsmakers, M. Stroomer, and W. Timmers, "Polarization conversion system LCD projection," in Proc. 13th Int. Display Res. Conf., Strasbourg, France, 1993, p. 253.

[13] H. S. Kwok and H. C. Huang, "Optical systems for liquid crystal display projectors," U.S. Patent 6793344 B2, Sep. 21, 2004.

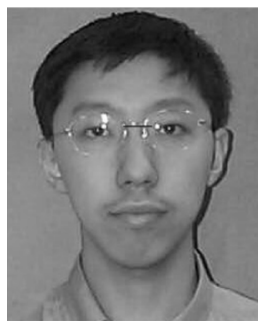

Xing-Jie Yu received the B.S. degree from Nankai University, Tianjin, China, in 2000, and the Ph.D. degree from Hong Kong University of Science and Technology, Hong Kong, in 2004.

His research interests and projects are LED illumination system, key optical components related to LCOS systems, color liquid crystal displays (LCDs), and bistable LCDs. He now is a Senior Display Technology Engineer with Philips Mobile Display Systems, Hong Kong.

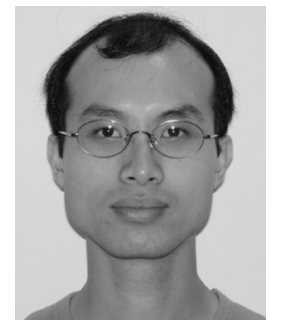

Y. L. Ho received the B.S. degree in engineering physics from the Hong Kong Polytechnic University, Hong Kong, in 1993, and the M.Phil. degree in physics and the M.Sc. degree in electronic engineering from the Hong Kong University of Science and Technology (HKUST), Hong Kong, in 1995 and 1999, respectively.

$\mathrm{He}$ is currently a Senior Technician with the Department of Electronic Engineering, HKUST.

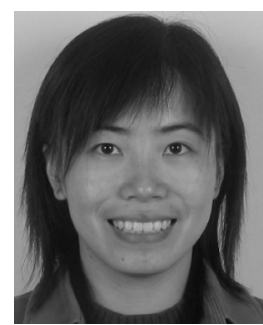

L. Tan received the B.S. degree from Beijing Normal University, Beijing, China, in 2001, and the M.S. degree from Zhejiang University, Hangzhou, China, in 2004. She is currently working toward the Ph.D. degree at the Department of Electrical and Electronic Engineering, Hong Kong University of Science and Technology.

From 2004 to 2005, she was an Optical Engineer with Asia Optical (Hangzhou) Innotek Company, Ltd., Hangzhou, China. Her research interests include fast response liquid crystal display (LCD) and wide-viewing-angle $\mathrm{LCD}$.

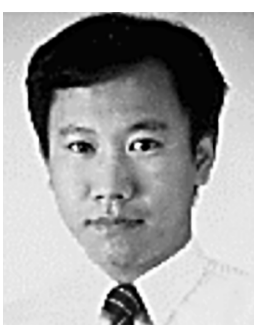

Ho-Chi Huang received the B.S. and M.S. degrees from National Taiwan University, Taipei, Taiwan, R.O.C., in 1982 and 1984, respectively, and the $\mathrm{Ph} . \mathrm{D}$. degree from the University of Washington, Seattle, in 1991, all in electrical engineering.

He joined the Department of Electrical and Electronic Engineering, Hong Kong University of Science and Technology, in 1991 and became an Associate Professor in 1999.

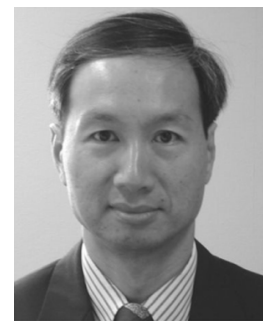

Hoi-Sing Kwok (S'73-M'78-SM'84-F'01) received the Ph.D. degree in applied physics from Harvard University, Cambridge, MA, in 1978.

$\mathrm{He}$ was a Professor of Electrical and Computer Engineering with the State University of New York, Buffalo, from 1980 to 1992 . He joined the Hong Kong University of Science and Technology, Hong Kong, in 1992. He has published over 500 papers and holds 25 patents in optics and liquid crystal display technologies.

Dr. Kwok is a Fellow of the Optical Society of America and the Society for Information Display. He was the recipient of the U.S. Presidential Young Investigator Award in 1984. 